\title{
Notizen und Kurzbeiträge
}

https://doi.org/10.1515/bd-2022-0011

\section{Literarischer Nachlass von Leo Perutz jetzt weltweit digital zugänglich}

\section{Deutsches Exilarchiv 1933-1945 ermöglicht Zugriff auf Manuskripte und bisher unveröffentlichte Familienkorrespondenz des Schriftstellers}

Zum Geburtstag des österreichischen Schriftstellers Leo Perutz am 2. November macht das Deutsche Exilarchiv 1933-1945 breite Teile seines digitalisierten Nachlasses online zugänglich.

Leo Perutz (1882-1957) war zu seiner Zeit einer der erfolgreichsten und meistgelesenen österreichischen Schriftsteller, bis er nach der Annexion Österreichs das Land im Juli 1938 verlassen musste und über Italien nach Palästina auswanderte. In Palästina bestritt er seinen Lebensunterhalt als Versicherungsmathematiker, ein Beruf, in dem Perutz auch zuvor schon gearbeitet und geforscht hatte. Ein schriftstellerischer Erfolg blieb nach seiner Flucht ins Exil weitgehend aus.

Seine fast durchgängig vorhanden kalendarischen Tagebuchaufzeichnungen (1909-1957) verfasste Perutz in der um 1900 populären sogenannten Gabelsberger Kurzschrift. Die Einträge geben Einblick in die literarische Produktion Perutz und politische Ereignisse. So nahm Perutz in seinem Tagebuch schon am 3. März 1938, 10 Tage vor dem Ereignis, die Annexion Österreichs mit den Worten „Finis Austriae" vorweg.

Perutz' umfangreiches Oeuvre wurde seit Ende der 1980er Jahre wiederentdeckt und neu aufgelegt. Es umfasst Novellen, Erzählungen, Theaterstücke, Reiseberichte, Dramen und Romane. Neben historischen Romanen wie „Die dritte Kugel“ (1915), „Der Marques de Bolibar“ (1920) und „Turlupin“ (1924) schrieb Perutz auch Unterhaltungsromane, wie „Zwischen neun und neun“ (1918), „Der Meister des Jüngsten Tages“ (1923) sowie den Bestseller „Wohin rollst Du, Äpfelchen“ (1928), von dem Ian Flemming, der berühmte James-Bond Autor schrieb: „Das Wort Genie hat längst durch Missbrauch an Wert und Sinn verloren, sonst hätte ich das Buch als einfach genial bezeichnet.“ 
Die beiden letzten Werke an denen er in Israel arbeitete, erschienen erst kurz vor seinem Tod bzw. posthum: „Nachts unter der steinernen Brücke“ (1957), „Der Judas des Leonardo“ (1959).

Sein Nachlass, der sich seit 1986 im Exilarchiv der Deutschen Nationalbibliothek befindet, umfasst Manuskripte $\mathrm{zu}$ fast allen Romanen und auch zu bislang unveröffentlichten bzw. unvollendeten Werken. Hinzu kommt eine umfangreiche Korrespondenz, darunter bislang noch unveröffentlichte Familienbriefe. Mit den jetzt weltweit zugänglichen Digitalisaten und Archivalien, darunter auch den Tagebüchern, bietet der Nachlass von Leo Perutz zahlreiche Anknüpfungspunkte für die Wissenschaft, sich seinem Werk und seiner Biografie zu nähern und neue Perspektiven auf Leo Perutz zu entwickeln.

\section{Digitalisierter Nachlass von Leo Perutz}

Online verfügbare Digitalisate im Katalog der Deutschen Nationalbibliothek: https://portal.dnb.de/opac/moveDown?currentResultId=partOf\%3D982013469 \%26any\%26online\&categoryId=dnb.dea.archivalien

\section{Ansprechpartnerin}

Dr. Sylvia Asmus, Leiterin des Deutschen Exilarchivs 1933-1945

Tel.: 069 1525-1900 | E-Mail: s.asmus@dnb.de

\section{Bilder}

Bildmaterial zur redaktionellen Verwendung im Zusammenhang mit Berichterstattung unter www.dnb.de/perutz

\section{Hintergrund}

Das Deutsche Exilarchiv 1933-1945 der Deutschen Nationalbibliothek hat die Aufgabe, sowohl die Veröffentlichungen als auch Archivmaterial des deutschsprachigen Exils während der Zeit der NS-Diktatur zu sammeln. Zu den Veröffentlichungen gehören alle zwischen 1933 und 1950 von deutschsprachigen Emigrant*innen im Ausland veröffentlichten Bücher und Broschüren aus den Bereichen Literatur, Politik, Wissenschaft und jüdische Emigration, außerdem die von ihnen herausgegebenen Zeitschriften. $\mathrm{Zu}$ den Archivalien gehören persönliche Nachlässe deutschsprachiger Emigrant*innen unterschiedlicher Disziplinen und Berufsgruppen sowie Archive von Exilorganisationen und Einzelautografen.

Seit März 2018 präsentiert das Deutsche Exilarchiv ausgewählte Exponate der eigenen Sammlung in der Dauerausstellung „Exil. Erfahrung und Zeugnis“. Zudem liegt die Federführung für das Netzwerkprojekt „Künste im Exil“ beim Deutschen Exilarchiv. 
In seinen Veranstaltungen und Wechselausstellungen nimmt das Exilarchiv weitere Themen in den Blick und zieht so Verbindungslinien zwischen dem historischen Exil und aktuellen Phänomenen.

\section{Goethe VR. Goethes Faust als interaktive Virtual Reality Experience ab 19. Oktober 2021 in der Deutschen Nationalbibliothek}

Ein Klassiker der deutschen Literatur virtuell: Mit „Goethe VR“ bringt die Deutsche Nationalbibliothek ab 19. Oktober 2021 Goethes Faust I und II vom Buch in eine Virtual-Reality-Anwendung. Gespielt werden kann an beiden Standorten in Leipzig und Frankfurt am Main.

„Goethe VR“ ist eine interaktive Virtual-Reality-Experience, die Johann Wolfgang von Goethes Tragödie „Faust I und Faust II“ für eine digitale Welt adaptiert. Eines der bekanntesten literarischen Werke wird damit ganz neu erlebbar. Den literarischen Klassiker als VR-Experience in der Deutschen Nationalbibliothek zu zeigen, ist zugleich ein Verweis auf den Sammelauftrag. Hier werden Werke in vielen Fassungen gesammelt: als Comics, als Kinderbuchbearbeitung, in Übersetzungen. Fast 3.000 verschiedene Ausgaben von Goethes Faust I und II sind Teil des Bestandes.

Ausgestattet mit VR-Brille und weiteren Tools sind Spieler`innen aktiv in die Erzählung eingebunden: Sie unterzeichnen den Pakt mit Mephisto, reisen in die Hexenküche, treffen Gretchen und suchen des Pudels Kern. Um alle auf eine sowohl literarische als auch emotionale Reise mitzunehmen, ist in sieben Szenen eine kontinuierliche Handlung mit eigenem Spannungsbogen erlebbar. Dabei sind Nähe zum Originaltext und die Einbindung der Originalsprache wichtiger Bestandteil der Adaption.

So wird eine virtuelle Welt erschaffen, die atmosphärisch und inhaltlich dem literarischen Vorbild entspricht und den Spieler*innen das Thema des literarischen Werks auf visuell ansprechende Art erfahrbar macht. Die Mitspielenden sollen die Konflikte, die zentralen Figuren und Leitmotive kennenlernen und Wissenswertes über den Autor und die Entstehung des Textes vermittelt bekommen.

Inhaltlich konzentriert die VR sich auf zeitgenössische Aspekte und greift Passagen auf, die als Reflexion über den modernen Menschen gelesen werden können. Fausts unerbittliche Suche nach dem Neuen, seine Rastlosigkeit und der Wunsch, den einen perfekten Moment zu erleben, bilden den Rahmen dieser digi- 
talen Faust-Fassung. Auch Goethes skeptischer Blick auf den zu seiner Zeit entstehenden Kapitalismus spielt in der Adaption eine Rolle: Je mehr der Mensch besitzen will, desto mehr zerstört er. „Goethe VR“ spielt in einer Wüstenlandschaft, deren Ästhetik an die Malerei des Surrealismus angelehnt ist. Die minimalistische Weite der Wüste setzt dabei Gegenstände in Szene und verweist zugleich auf die Ausbeutung der Natur durch den Menschen, die in der gewählten Interpretation eine zentrale Rolle spielt.

Die VR ist in neun Sprachen verfügbar: Deutsch, Englisch, Portugiesisch, Spanisch, Russisch, Indonesisch, Polnisch, Tschechisch und Arabisch. Gespielt werden kann „Goethe VR“ ab 19. Oktober 2021 kostenlos während der Öffnungszeiten des Deutschen Buch- und Schriftmuseums Leipzig (Dauerausstellung) und im Foyer der Deutschen Nationalbibliothek in Frankfurt am Main. Spieldauer: ca. $15 \mathrm{~min}$.

Eröffnet wird „Goethe VR“ im Rahmen einer Preview am Sonntag, 17. Oktober, 10-16 Uhr in Anwesenheit der Spiele-Designer`innen und weiteren Projektverantwortlichen von ZDF Digital im Deutschen Buch- und Schriftmuseum Leipzig. Eine Produktion von ZDF Digital in Kooperation mit dem Deutschen Buch- und Schriftmuseum der Deutschen Nationalbibliothek, den Goethe-Instituten sowie dem Goethe-Museum Düsseldorf, gefördert von der Computerspieleförderung des Bundes.

\section{Goethe VR. Goethes Faust als interaktive Virtual Reality Experience}

- In Leipzig: Dienstag bis Sonntag 10-18 Uhr

Donnerstag 10-20 Uhr

An Feiertagen 10-18 Uhr

Montag geschlossen

Eintritt frei

- In Frankfurt: Montag bis Freitag 9-21:30 Uhr

Samstag 10-17:30 Uhr

An Sonn- und Feiertagen geschlossen, außerdem in der Zeit von 24. Dezember 2021 bis 2. Januar 2022

Eintritt frei

\section{Weitere Informationen im Internet:}

- Website zur Ausstellung: www.dnb.de/goethevr

- Bildmaterial für die Berichterstattung: www.dnb.de/presse

\section{Ansprechpartnerin Deutsches Buch- und Schriftmuseum:}

Dr. Stephanie Jacobs

Tel.: +49 341 2271-575 | E-Mail: s.jacobs@dnb.de 


\section{Hintergrund}

Das Buch hat wie kein anderes Medium unsere Kultur und Zivilisation geprägt: Seit Jahrhunderten wird unser Wissen über die Welt und über den Menschen in Büchern gespeichert. Die Sammlung, Ausstellung und wissenschaftliche Bearbeitung buch- und mediengeschichtlicher Zeugnisse ist die Aufgabe des Deutschen Buch- und Schriftmuseums der Deutschen Nationalbibliothek. 1884 als Deutsches Buchgewerbemuseum in Leipzig gegründet gilt es als das weltweit älteste und nach Umfang und Qualität der Bestände als eines der bedeutendsten Museen auf dem Gebiet der Buchkultur. Zur Sammlung gehören neben Handschriften, historischen Drucken, Buntpapieren und moderner Buchkunst ebenso Archivalien und Nachlässe zur Schrift- und Typografiegeschichte. Zusammen mit der weltweit größten Wasserzeichensammlung sowie Schreibgeräten und Maschinen zur Buch- und Papierherstellung erlauben die Bestände des Museums ein interdisziplinäres Herangehen an kultur- und medienwissenschaftliche Fragestellungen.

\section{Millionen Netzpublikationen}

Mehr als 10 Millionen Netzpublikationen (NP) bietet die Deutsche Nationalbibliothek zur Nutzung an, täglich werden es mehr: Inzwischen stellt der Zugang an Netzpublikationen rund 60 Prozent des gesamten Zugangs. Zwei Drittel des NP-Bestandes besteht aus periodisch erscheinenden Netzpublikationen (E-Journals und E-Paper) und rund ein Drittel aus Monografien (Hochschulschriften, Books on Demand, Musikalien, E-Books und Hörbücher). Der Gesamtbestand der Deutschen Nationalbibliothek beträgt mehr als 42 Millionen Medieneinheiten. Knapp ein Viertel aller Bestände der Deutschen Nationalbibliothek ist also mittlerweile digital. Dieses Verhältnis wird sich in den nächsten Jahrzehnten immer weiter zugunsten der Netzpublikationen verschieben.

„10 Millionen Netzpublikationen, davon rund die Hälfte in den letzten drei Jahren - das ist Rückenwind für eine zentrale strategische Priorität der Deutschen Nationalbibliothek: Wir wollen unsere digitalen Sammlungen weiter ausbauen“, so Frank Scholze, der Generaldirektor der Deutsche Nationalbibliothek.

Ob auf Papier oder anderen Trägermaterialien oder in digitaler Form: Die Deutsche Nationalbibliothek sammelt Bücher, Zeitschriften, Karten, Noten, Musik und vieles andere mehr - ohne Wertung, im Original und möglichst lückenlos. Um ihre Gedächtnisfunktion auch für neue Publikationsformate zu erfüllen, baut sie die digitalen Sammlungen aus und entwickelt attraktive und nutzergerechte 
Präsentationsformen. Mit ihren Diensten und Angeboten fördert sie die Informations- und Meinungsfreiheit und stärkt zentrale Werte unserer Demokratie.

Seit 2006 erstreckt sich der gesetzliche Sammelauftrag der Deutschen Nationalbibliothek auch auf Online-Publikationen, die sogenannten Netzpublikationen. Die Ablieferung der Netzpublikationen erfolgt über automatisierte Verfahren. Dabei werden die Metadaten in anerkannten internationalen Standards gemeinsam mit der digitalen Publikation übermittelt. Der Ablieferungsprozess der Medienwerke unterliegt einer maschinellen Qualitätskontrolle, bevor die Metadaten schließlich in den Katalog und die Volltexte in ein zertifiziertes Langzeitarchiv eingespielt werden, um dort „für die Ewigkeit“ bewahrt zu werden. Ob eine Publikation nur in den Lesesälen der Deutschen Nationalbibliothek genutzt werden kann oder im Web frei zugänglich ist, steuern die Abliefernden durch die Angabe von Zugriffsrechten in den Metadaten.

Ein Vorteil der Pflichtablieferung für die Verlage ist die Langzeitarchivierung ihrer E-Books und E-Journals durch die Deutsche Nationalbibliothek. Durch die Verzeichnung im eigenen und in kooperierenden Katalogen sind die Publikationen besser sichtbar; es kann ein Link auf die Homepage des Verlags mitgegeben werden und gegebenenfalls können Verlage auch von der Konvertierung ihrer mitgelieferten Metadaten nach MARC-XML profitieren.

In den „Digital Humanities“ bündelt und entfaltet sich seit einigen Jahren schwerpunktmäßig der „Digitale Wandel“ in den Geisteswissenschaften. Als eine der großen Gedächtnisinstitutionen Deutschlands bietet die Deutsche Nationalbibliothek ihre Datenbestände und digitalen Sammlungen in ihren Lesesälen für Wissenschaft und Forschung sowie für experimentelles und kreatives Arbeiten so weitgehend wie möglich an. Damit leistet sie ihren Beitrag dazu, dem archivierten Kulturerbe Deutschlands einen lebendigen Platz in der Gesellschaft zu sichern. Die neben der Sammlung physischer Medienwerke entstandene umfangreiche Sammlung an E-Books, E-Journals, E-Paper und anderen digitalen Objekten ist nach den Vorgaben des Urhebergesetzes auch für eine automatisierte Analyse (Text und Data Mining) für wissenschaftliche nicht-kommerzielle Zwecke nutzbar.

\section{Weitere Informationen im Internet:}

- Website „Sammlung unkörperlicher Medienwerke“: https://www.dnb.de/ netzpublikationen

\section{Hintergrund}

Die Deutsche Nationalbibliothek sammelt seit über 100 Jahren alle Inlandspublikationen in Schrift, Bild und Ton sowie weltweit deutschsprachige Veröffentlichungen und solche mit Bezug zu Deutschland ab 1913, dokumentiert und archiviert sie und macht sie der Öffentlichkeit zugänglich. Ihre umfassenden 
Dienstleistungen bietet sie an den beiden Standorten in Leipzig und Frankfurt am Main und im urheberrechtlich zulässigen Rahmen in digitaler Form global an.

Mit dem Deutschen Exilarchiv 1933-1945 und dem Deutschen Buch- und Schriftmuseum verfügt die Deutsche Nationalbibliothek darüber hinaus über wertvolle und reichhaltige Sondersammlungen. Durch Lesungen, Ausstellungen, Vorträge und Konzerte macht sie regelmäßig auf ihre Schätze aufmerksam und fördert Buchkultur, Lesekultur und Musikkultur mit mehr als 42 Millionen Medieneinheiten, jährlich rund 220.000 Besucher`innen an den beiden Standorten Leipzig und Frankfurt am Main und mit einem bunten und hochkarätigen Veranstaltungsprogramm.

\section{Kontakt:}

Stephan Jockel

Deutsche Nationalbibliothek

Strategische Entwicklungen und Kommunikation | Pressesprecher

Adickesallee 1 | 60322 Frankfurt am Main

Tel.: +4969 1525-1005 | E-Mail: s.jockel@dnb.de

\section{Schulteam entwickelt App für bessere Vereinbarkeit von Beruf und Familie}

\section{Schüler*innen aus Gelsenkirchen schaffen es mit App-Idee auf den ersten Platz beim internationalen YES! - Young Economic Summit}

Vom 22. bis 24. September 2021 kamen digital zehn Schulteams aus Deutschland und sieben weltweite Teams aus Hong Kong, der Schweiz, Großbritannien und aus den USA zum internationalen Finale des YES! - Young Economic Summit zusammen. YES! ist der größte Schulwettbewerb für Wirtschaft und Gesellschaft in Deutschland. Dieses Jahr arbeiteten 700 Schüler`innen in 71 Schulteams sechs Monate an Lösungsansätzen. Beim ersten internationalen Finale des YES! gewann das Team vom Max-Planck-Gymnasium Gelsenkirchen mit einer App, mit der Arbeitnehmer^innen die Familienfreundlichkeit ihres Unternehmens bewerten können.

„Wer Beruf und Familie vereinbaren will, braucht ein Unternehmen, das dabei mitspielt. Unsere Lösung dafür ist die App KINT. In unserer App können Beschäftigte ihre Arbeitgeber transparent nach familienfreundlichen Aspekten 
bewerten. Bewerber*innen können so in einem Ranking sehen, welche Unternehmen für ihr Lebensmodell interessant sind. “So erklären die Schüler^innen vom Max-Planck-Gymnasium Gelsenkirchen ihre Lösungsidee gegen sinkende Geburtenraten und eine Überlastung des Rentensystems.

Sie erläutern: „Es war für uns eine großartige Erfahrung, am YES! teilzunehmen. Wir hoffen, dass wir andere junge Menschen inspirieren können, sich bei der Lösung gesellschaftlicher Herausforderungen einzubringen. Wir wünschen uns, dass unsere Idee nachhaltig zur Verbesserung der Vereinbarkeit von Familie und Beruf beitragen kann.“

Dr. Daniel Kamhöfer (Düsseldorf Institute for Competition Economics [DICE]) und Dr. Matthias Westphal (RWI - Leibniz-Institut für Wirtschaftsforschung) betreuten das Team aus Gelsenkirchen als wissenschaftliche Partner.

Ihr Statement: „Eine super Leistung der Schülerinnen und Schüler! Nicht nur der Sieg beim Wettbewerb ist ein großer Erfolg, sondern auch die Idee, die den Sieg gebracht hat. Eine App, die es erlaubt, Unternehmen nach ihrer Familienfreundlichkeit zu bewerten, ist ein sehr konkreter Ansatz, mit dem es gelingen kann, die Vereinbarkeit von Familie und Beruf zu erhöhen: Angehende Eltern können informierte Berufsentscheidungen treffen und Firmen haben einen Anreiz, ihre Familienfreundlichkeit weiter zu verbessern. Besonders beeindruckt hat uns auch, wie souverän die Schüler*innen das für sie doch noch sehr weit in der Zukunft liegende und deshalb abstrakte Thema Familiengründung vor Fachexpert*innen präsentiert haben.“

Über ein halbes Jahr hat sich die Projektgruppe aus Nordrhein-Westfalen auf das Thema vorbereitet, unterstützt durch die Expert*innen der ZBW - Leibniz-Informationszentrum Wirtschaft, des Düsseldorf Institute for Competition Economics (DICE) und des RWI - Leibniz-Institut für Wirtschaftsforschung. Die feierliche Preisverleihung findet, begleitet durch das Bundesministerium für Wirtschaft und Energie, digital statt.

Platz 2 erreichte das Annette-Kolb-Gymnasium Traunstein aus Bayern mit seiner Idee „Get ToGether to get better!“”, einem Lösungsvorschlag für bessere Finanzkompetenz unter Geflüchteten und Menschen ohne Fluchterfahrung in Deutschland. Auf den 3. Platz schaffte es das Albert-Einstein-Gymnasium Frankenthal aus Rheinland-Pfalz mit seiner Idee „Come together“ - auch dies eine Idee für die bessere Integration von Geflüchteten, die jedoch eher spielerische Aspekte betont. Der Sonderpreis YES! International Award ging an die South Forsyth High School, Cummings, Georgia, USA.

Der YES!-Jurypreis für „Best Scientific Analysis“ ging 2021 an das Landschulheim Grovesmühle in Sachsen-Anhalt und an die Deutsche Berufsschule Hong Kong. Beide Schulteams erhalten jetzt die Möglichkeit, ihre Lösungsidee in der Online- und Print-Ausgabe der Fachzeitschrift „Wirtschaftsdienst“ wissenschaft- 
lich zu publizieren. Begleitende Forschende sind Mitautor^innen, entweder federführend oder begleitend.

Das „YES! - Young Economic Summit“ ist seit 2015 der größte Schulwettbewerb rund um wirtschaftliche und gesellschaftliche Herausforderungen für die Generation Z. 2021 diskutierten die Schulteams aus der ganzen Bundesrepublik zusammen mit sieben Schulteams aus Europa, Asien und Amerika ihre Lösungsansätze und entschieden am Ende selbst, wer gewinnt.

Beim ersten internationalen Finale des YES! 2021 diskutierten 150 Jugendliche ihre Ideen mit Gästen aus aller Welt. Expert^innen aus Australien, Belgien, Deutschland, Frankreich, den Niederlanden, Österreich, Portugal, der Schweiz, Großbritannien und den USA waren dabei. Dazu zählten zum Beispiel Staatssekretärin Juliane Seifert, Bundesministerium für Familie, Senioren, Frauen und Jugend; Christoph Heinrich, Vorstand Naturschutz des WWF Deutschland; Sami Kanaan, Stadtpräsident von Genf oder Estelle Göger, Mitglied des Kabinetts des Kommissars für Wirtschaft bei der Europäischen Kommission.

\section{Weitere Informationen im Internet:}

- Lösungsidee „Make Your Job KINTeresting“: https://www.young-economicsummit.org/max-planck-gymnasium-gelsenkirchen-2021

- Alle Infos zu den Gewinner-Teams: https://www.young-economic-summit. org/yes_2021

\section{Über das YES!}

Das „YES! - Young Economic Summit“ ist einer der größten Schulwettbewerbe rund um wirtschaftliche und gesellschaftliche Herausforderungen für die junge Generation. Schüler^innen erarbeiten eigenständig Lösungen für globale ökonomische, ökologische und gesellschaftliche Herausforderungen und präsentieren diese im Rahmen eines Schulkongresses, dem „YES! - Young Economic Summit“ in englischer Sprache. Im Diskurs mit Führungspersönlichkeiten und anderen Schüler*innen schärfen sie ihren Blick auf globale Zusammenhänge und entwickeln eigene innovative Lösungsvorschläge. Über die besten Lösungen wird demokratisch abgestimmt. Die gewählten Lösungen werden ausgezeichnet und öffentlichkeitswirksam zur Umsetzung an geeignete Adressaten übergeben und nachverfolgt. Auf dem Weg zum YES! werden alle teilnehmenden Schulen kontinuierlich zu den Themen Recherche, Forschung, Präsentation und Medienarbeit unterstützt und begleitet. Damit nimmt das YES! Empfehlungen der Kultusministerkonferenz auf, Schüler*innen darin zu befähigen, Medienanwendungen kritisch einzusetzen. Das „YES! - Young Economic Summit“ steht unter der Schirmherrschaft des Bundesministeriums für Wirtschaft und Energie und ist ein gemeinsames Projekt der ZBW - Leibniz-Informationszentrum Wirtschaft und der 
Joachim Herz Stiftung. 2020 wurde das YES! ausgezeichnet mit dem BMBF-Förderpreis „Raising the Profile of Education and Science Diplomacy“. 2021 startete auch der Prototyp YES! MINT - eine Beteiligung der Klassenstufen 5 bis 9 an der Schnittstelle zwischen MINT und Wirtschaft.

URL: www.young-economic-summit.org

\section{Über die ZBW - Leibniz-Informationszentrum Wirtschaft}

Die ZBW - Leibniz-Informationszentrum Wirtschaft (ZBW) ist die weltweit größte Informationsinfrastruktur für die Wirtschaftswissenschaften. Die Einrichtung beherbergt rund 4 Millionen Medieneinheiten und ermöglicht den Zugang zu Millionen wirtschaftswissenschaftlicher Online-Dokumente. Allein 2020 wurden mehr als 12 Millionen digitale Volltexte heruntergeladen. Daneben stellt die ZBW eine rasant wachsende Sammlung von Open-Access-Dokumenten zur Verfügung. EconStor, der digitale Publikationsserver, verfügt aktuell über 220.000 frei zugängliche Aufsätze und Working Papers. Mit EconBiz, dem Fachportal für wirtschaftswissenschaftliche Fachinformationen, können Studierende oder Forschende in über 10 Millionen Datensätzen recherchieren. Zudem gibt die ZBW die beiden wirtschaftspolitischen Zeitschriften Wirtschaftsdienst und Intereconomics im Gold Open Access heraus. Die ZBW ist eine forschungsbasierte wissenschaftliche Bibliothek. Mit Professuren in der Informatik, Wirtschaftswissenschaft und Medienwissenschaft und deren international besetzter Doktorandengruppe beschäftigt sich die ZBW transdisziplinär mit dem Thema Open Science. Die ZBW ist in ihrer Forschung international vernetzt. Hauptsächliche Kooperationspartner kommen aus EU-Vorhaben, aus DFG- bzw. BMBF-Projekten sowie aus dem Leibniz-Forschungsverbund Open Science. Die ZBW ist Teil der Leibniz-Gemeinschaft und Stiftung des öffentlichen Rechts. Sie wurde mehrfach für ihre innovative Bibliotheksarbeit mit dem internationalen LIBER Award ausgezeichnet.

URL: www.zbw.eu

\section{Über die Joachim Herz Stiftung}

Die gemeinnützige Joachim Herz Stiftung arbeitet überwiegend operativ und ist vorrangig in den Themenfeldern Naturwissenschaften, Wirtschaft sowie Persönlichkeitsbildung tätig. In diesen drei Bereichen werden auch kleine, innovative Projekte Dritter unterstützt. Zudem fördert die Stiftung Forschungsprojekte in den Themenfeldern Medizin, Recht und Ingenieurwissenschaften sowie Vorhaben im deutsch-amerikanischen Austausch. Die Joachim Herz Stiftung wurde 2008 errichtet und gehört zu den großen deutschen Stiftungen.

URL: www.joachim-herz-stiftung.de 


\section{Pressekontakt}

Dr. Doreen Siegfried, Pressesprecherin

ZBW - Leibniz-Informationszentrum Wirtschaft

Düsternbrooker Weg 120 | 24105 Kiel

Tel.: 043188 14-455 | E-Mail: d.siegfried@zbw.eu

URL: www.zbw.eu

\section{Volker Hinz goes BSB: Die Bayerische Staatsbibliothek erwirbt das fotografische Gesamtwerk von Volker Hinz}

Die Bayerische Staatsbibliothek hat das Fotoarchiv von Volker Hinz (1947-2019) mit rund 1,3 Millionen Aufnahmen erworben. Es umfasst das gesamte fotografische Lebenswerk von Volker Hinz für das Wochenmagazin stern sowie eine Vielzahl freier Arbeiten. Mit diesem herausragenden Zeugnis der Fotografie Deutschlands baut die Bibliothek ihr umfangreiches Bildarchiv, das größte in öffentlicher Hand in Deutschland, weiter aus.

Das analoge Archiv von Volker Hinz besteht aus Schwarz-weiß- und Farbnegativen, Dias und Fine Art Prints. Ergänzend dazu übernimmt die Bayerische Staatsbibliothek die umfangreiche Fotobibliothek des Fotografen. Die Bayerische Staatsbibliothek wird dieses einzigartige Archiv als Ganzes dauerhaft bewahren, Schritt für Schritt digitalisieren und weltweit zugänglich machen.

Der 1947 in Hamburg-Blankenese geborene Volker Hinz wurde mit 24 Jahren Leiter der „Sven Simon Bildagentur“ in Bonn, 1974 kam er zum Wochenmagazin stern nach Hamburg. Für die Zeitschrift arbeitete Volker Hinz mit kurzer Unterbrechung 38 Jahre - davon acht Jahre in New York. Seine zahlreichen Bildreportagen für den stern, insbesondere seine viel beachteten Portraits berühmter Persönlichkeiten machten Volker Hinz weit über Deutschland hinaus bekannt. Viele seiner Bilder sind heute Teil des kollektiven Gedächtnisses seiner Zeit. „Der Fotograf“ - so das Credo von Volker Hinz - „darf nicht langweilen. Langweilige Bilder gibt es genug."

Dr. Klaus Ceynowa, Generaldirektor der Bayerischen Staatsbibliothek: „Wir sind stolz und glücklich, das fotografische Werk von Volker Hinz, das seine Arbeiten für den stern umfasst und zugleich weit darüber hinausgeht, jetzt für unser Bildarchiv erwerben zu können. Dies unterstreicht nachdrücklich den Anspruch der Bibliothek, eines der europaweit bedeutendsten zeitgeschichtlichen Foto- 
archive Europas zu kuratieren. Durch seine sukzessive Digitalisierung soll dieser Bilderkosmos für die künftige weltweite Sichtbarkeit und Nutzung aktiviert werden.“

\section{Zum Bildarchiv der Bayerischen Staatsbibliothek}

Das Bildarchiv der Bayerischen Staatsbibliothek umfasst mehr als 18 Millionen vorwiegend dokumentarische Aufnahmen mit zeitgeschichtlichem Schwerpunkt. Es handelt sich damit um das größte Bildarchiv in öffentlicher Hand in Deutschland. Den Hauptfonds macht das 2019 übernommene Fotoarchiv des Magazins stern mit mehr als 15 Millionen Bildern aus. Der Bestand wird in den nächsten Jahren sukzessive digitalisiert.

URL: https://www.bsb-muenchen.de/sammlungen/bilder/fotoarchive

\section{Über die Bayerische Staatsbibliothek}

Die Bayerische Staatsbibliothek, gegründet 1558 durch Herzog Albrecht V., genießt als internationale Forschungsbibliothek Weltrang. Mit rund 34,5 Millionen Medieneinheiten ist sie die größte Universalbibliothek Deutschlands und gehört zu den bedeutendsten Gedächtnisinstitutionen der Welt. Mit rund 2,7 Millionen digitalisierten Titeln verfügt die Bayerische Staatsbibliothek über den größten digitalen Datenbestand aller deutschen Bibliotheken. Die Bibliothek bietet vielfältige Dienste im Bereich innovativer digitaler Nutzungsszenarien an.

Bayerische Staatsbibliothek | Ludwigstr. 16, 80539 München | www.bsbmuenchen.de

\section{Bildmaterial}

URL: https://syncandshare.lrz.de/getlink/fi46pH1XAHwKCKDYYNSPGBMT Copyright / Alle Rechte: Bayerische Staatsbibliothek/Bildarchiv/Volker Hinz

\section{Ansprechpartner}

Dr. Klaus Ceynowa, Generaldirektor

Tel.: +49 89 28638-2201 | E-Mail: direktion@bsb-muenchen.de

Ulrike Rehusch, Presse- und Öffentlichkeitsarbeit

Tel.: +49 89 28638-2057 | E-Mail: presse@bsb-muenchen.de 


\section{Historische Zeitungen online: Bayerische Staatsbibliothek baut ihr Zeitungsportal digiPress auf 8,2 Millionen Seiten aus}

Die Bayerische Staatsbibliothek erweitert erneut ihr digitales Angebot: Mit der Bereitstellung von weiteren mehr als 361.000 Zeitungsseiten sind nun insgesamt rund 8,2 Millionen Zeitungsseiten in digiPress aufrufbar. Im Portal digiPress werden alle digitalisierten historischen Zeitungen unterschiedlichster Regionen und Sprachen vom 17. bis ins 20. Jahrhundert aus dem Bestand der Bayerischen Staatsbibliothek mit einer Fülle an Funktionalitäten zentral präsentiert.

In Umfang und Service ist digiPress derzeit das mit weitem Abstand größte kulturelle und wissenschaftliche Angebot dieser Art in Deutschland. Mit der für Frühjahr 2022 geplanten Bereitstellung von rund 33.000 Ausgaben der Münchner Neuesten Nachrichten von 1875 bis 1932 wird das Portal eine weitere zentrale historische Quelle präsentieren und auf circa 8,5 Millionen Seiten anwachsen.

Sämtliche Titel sind einzeln oder übergreifend im Volltext durchsuchbar. Die Suchergebnisse können nach Titel, Sprache, Erscheinungszeitraum und Erscheinungsort gefiltert werden. Neben der Volltextsuche ermöglicht eine Kalenderübersicht einen datumsorientierten, titelübergreifenden Einstieg für das Blättern in den Ausgaben. Die Titel sind zudem alphabetisch sowie nach Verbreitungsort sortierbar. Die Digitalisate lassen sich komfortabel und stufenlos vergrößern und sind so besonders gut lesbar. Dies ist auf den Einsatz des so genannten IIIF-Standards (International Image Interoperability Framework) zurückzuführen. IIIF ist heute der Maßstab für die Visualisierung, die Strukturierung und den Austausch digitaler Objekte zwischen Kulturinstitutionen aus aller Welt.

Die in digiPress präsentierten digitalisierten Zeitungen reichen vom 17. Jahrhundert bis vorwiegend in die 1920er Jahre. Schwerpunkt der in digiPress präsentierten Digitalisate sind bayerische Zeitungen ab dem 17. Jahrhundert, einzigartige Titel wie Revolutionszeitungen aller politischen Richtungen (1848/49), Parteizeitungen unterschiedlichster Couleur, Verbands- und Vereinszeitungen sowie humoristisch-satirische Blätter. Zahlreiche der in digiPress verfügbaren Zeitungen sind von überregionaler Bedeutung, wie z. B. die Allgemeine Zeitung/ Cotta'sche Zeitung oder ab Frühjahr 2022 die Münchner Neuesten Nachrichten.

Generaldirektor Klaus Ceynowa: „Zeitungen sind der ,Sekundenzeiger der Geschichte، und damit von hohem Interesse sowohl für die Wissenschaft wie auch allgemein für die interessierte Öffentlichkeit. Das Zeitungsportal digiPress ist ein Paradebeispiel dafür, wie digitalisierte Bestände schnell, unkompliziert und effizient durchsucht und die Ergebnisse von jedem Bürger genutzt werden können.“ 
Link zum Portal digiPress: https://digipress.digitale-sammlungen.de

Pressebilder finden Sie unter https://syncandshare.lrz.de/getlink/fiX3D1Cd W4hA9LaQ3x7PxRc5.

Bildnachweis: Bayerische Staatsbibliothek/Öffentlichkeitsarbeit

\section{Über die Bayerische Staatsbibliothek}

Die Bayerische Staatsbibliothek, gegründet 1558 durch Herzog Albrecht V., genießt als internationale Forschungsbibliothek Weltrang. Mit rund 34,5 Millionen Medieneinheiten ist sie die größte Universalbibliothek Deutschlands und gehört zu den bedeutendsten Gedächtnisinstitutionen der Welt. Mit rund 2,7 Millionen digitalisierten Titeln verfügt die Bayerische Staatsbibliothek über den größten digitalen Datenbestand aller deutschen Bibliotheken. Die Bibliothek bietet vielfältige Dienste im Bereich innovativer digitaler Nutzungsszenarien an.

Bayerische Staatsbibliothek | Ludwigstr. 16, 80539 München | www.bsbmuenchen.de

\section{Ansprechpartner}

Birgit Seiderer, Abteilung Bestandsentwicklung und Erschließung 2

Tel.: 089286382627 | E-Mail: zeitungen@bsb-muenchen.de

Ulrike Rehusch, Presse- und Öffentlichkeitsarbeit

Tel.: 089 28638-2057 | E-Mail: presse@bsb-muenchen.de

\section{„Facing the Balkans“ - Staatsbibliothek zeigt Fotoausstellung zu Südosteuropa}

Die Bayerische Staatsbibliothek widmet ihre diesjährige Jahresausstellung mit dem Titel „Facing the Balkans“ dem renommierten Fotojournalisten und ehemaligen stern-Fotografen Harald Schmitt. Von 11. November 2021 bis 4 . März 2022 sind im Fürstensaal und Prachttreppenhaus ausgewählte Fotografien seiner Südosteuropa-Reisen zu sehen, die dazu einladen, das eigene Balkanbild zu hinterfragen.

Über einhundert Fotografien aus elf Ländern, die während Schmitts fünf Reisen zwischen 2015 und 2021 entstanden, zeigen Südosteuropa von Slowenien im Norden bis Albanien im Süden, von Kroatien im Westen bis Transnistrien im Osten. Im Mittelpunkt der Aufnahmen stehen die Menschen, denen Harald Schmitt begegnet ist: Er fotografierte erfolgreiche Unternehmer*innen, Familien 
bei Hochzeiten und Festen, Tourist*innen, Gläubige oder Landbewohner bei der Feldarbeit. Eindrücklich thematisiert er auch das Schicksal der Flüchtlinge auf der sogenannten Balkanroute und dokumentiert die allgegenwärtigen Relikte der letzten Kriege und Orte der Erinnerung. In insgesamt sieben Themenbereichen (Reise, Flucht, Glaube, Erinnern, Wandel, Landleben, Verbundenheit) zeichnen die Fotografien Schmitts ein eindrucksstarkes Panorama der Regionen.

Harald Schmitt, geboren 1948, war von 1977 bis 2011 als Fotoreporter beim stern tätig und prägte in seiner über 50-jährigen Karriere das visuelle Gedächtnis Deutschlands mit. Für seine Arbeit erhielt er sechs World Press Photo Awards. Seit 2015 gilt Schmitts Interesse Regionen, die hierzulande vergleichsweise wenig Aufmerksamkeit erhalten: Ost- und Südosteuropa. Etwa 3.000 digitale Bilder von Reisen dorthin übereignete Schmitt der Bayerischen Staatsbibliothek. Sie ergänzen die etwa 100.000 Bilder von ihm, die mit der Schenkung des analogen Fotoarchivs des stern 2019 in die Bayerische Staatsbibliothek kamen.

Harald Schmitt: „Osteuropa ist ebenso nah wie unbekannt. Es wird Zeit, das zu ändern“.

„Die Fotoausstellung möchte dazu einladen, eine neue Perspektive auf Südosteuropa einzunehmen“, so Generaldirektor Dr. Klaus Ceynowa. „Es freut mich sehr, dass Harald Schmitt sich entschieden hat, die Ergebnisse seiner bemerkenswerten Fotoreisen gemeinsam mit der Bayerischen Staatsbibliothek in einer Ausstellung zu präsentieren.“

Der reich bebilderte Ausstellungskatalog (Kerber Verlag) bettet die Fotografien ein in spannende Essays von Wissenschaftler*innen des Leibniz-Instituts für Ost- und Südosteuropaforschung (IOS).

\section{November 2021 - 4. März 2022}

\section{Facing the Balkans. Südosteuropa in Fotografien von Harald Schmitt}

Montag bis Freitag 11 - 19 Uhr

An Wochenenden und Feiertagen, am 24. und 31. Dezember sowie am 1. März geschlossen

Eintritt frei

Informationen zur Ausstellung, zu Führungen und zum Begleitprogramm: http:// www.facing-the-balkans.de

\section{Katalog}

Erschienen im Kerber Verlag, erhältlich in der Ausstellung für 35,00 Euro.

Mit freundlicher Unterstützung der Förderer und Freunde der Bayerischen Staatsbibliothek e. V. 


\section{Ort}

Bayerische Staatsbibliothek, Ludwigstr. 16, 80539 München

U3/6, Bus 58/68/153/154 Haltestelle Universität

Bus 100/153 Haltestelle Von-der-Tann-Straße

\section{Fotos}

Druckfähige Pressebilder inkl. Copyright können Sie hier herunterladen: https:// syncandshare.lrz.de/getlink/fiWttewwSaZdy4F6CjoVf2H5

Fotonachweis / Alle Rechte: BSB/STERN-Fotoarchiv/Harald Schmitt

\section{Über die Bayerische Staatsbibliothek}

Die Bayerische Staatsbibliothek, gegründet 1558 durch Herzog Albrecht V., genießt als internationale Forschungsbibliothek Weltrang. Mit rund 34,5 Millionen Medieneinheiten ist sie die größte Universalbibliothek Deutschlands und gehört zu den bedeutendsten Gedächtnisinstitutionen der Welt. Mit rund 2,7 Millionen digitalisierten Titeln verfügt die Bayerische Staatsbibliothek über den größten digitalen Datenbestand aller deutschen Bibliotheken. Die Bibliothek bietet vielfältige Dienste im Bereich innovativer digitaler Nutzungsszenarien an.

Bayerische Staatsbibliothek|Ludwigstr. 16, 80539 München|www.bsb-muenchen. de

\section{Kontakt}

Peter Schnitzlein

Presse- und Öffentlichkeitsarbeit

Tel.: +49 89 28638-2429

E-Mail: presse@bsb-muenchen.de

\section{Von Alef Bet bis Zion - 17 Motive jüdischen Lebens}

\section{Ausstellungsprojekt der Frankfurter Universitätsbibliothek}

Die Universitätsbibliothek Johann Christian Senckenberg Frankfurt am Main beteiligt sich am Festjahr „1700 Jahre jüdisches Leben in Deutschland“. Das Seminar- und Ausstellungsprojekt der Bibliothek nähert sich entlang von 17 Motiven - Ereignissen, Praktiken und Texten - der vielstimmigen deutsch-jü- 
dischen Kultur in Vergangenheit und Gegenwart. Im Zentrum stehen Objekte der eigenen Hebraica- und Judaica-Sammlung, die zu den bedeutendsten ihrer Art in Europa zählt.

\section{Ausstellung „17 Motive jüdischen Lebens“:}

4. November 2021 - 16. Januar 2022 im

Schopenhauer-Studio der Universitätsbibliothek,

Bockenheimer Landstraße 134-138,

geöffnet dienstags bis sonntags, 13-18 Uhr

Die Zahl 17 steckt in Anlehnung an das Jubiläumsjahr den Rahmen, die einzelnen Motive geben einen mosaikhaften Einblick in deutsch-jüdisches Leben und sprechen dabei meist mehrere Aspekte an. „So geht es etwa bei ,Alef Bet‘ nicht nur um das hebräische Alphabet und damit die Grundlage aller jüdischen Sprachen, “wie Kerstin von der Krone, die Leiterin der Hebraica- und Judaica-Sammlung betont, „sondern auch um Mehrsprachigkeit und die damit verbundenen Praktiken des Übersetzens.“

Die von ihr und ihrem Team ausgewählten Motive waren die Grundlage für ein Projektseminar an der Goethe-Universität im Sommersemester 2021. Studierende unterschiedlicher Fächer haben die Sammlungsbestände der Universitätsbibliothek vor dem Hintergrund des Projektes erkundet, Objekte entdeckt und Objekterzählungen geschrieben. Schrittweise werden diese nun auf der Webseite des Projektes veröffentlicht und durch weitere Quellen, Bilder, Texte und Perspektiven ergänzt. So wird sich zum Ende des Festjahres das Mosaik der 17 Motive mit immer mehr Leben füllen. Judith Blume, Kerstin von der Krone und Johanna Weiler (alle UB Frankfurt) - die das Seminar gemeinsam leiteten und den Kern des Projektteams bilden - sind begeistert von dem Engagement und der Professionalität der Studierenden. „Es ist beeindruckend, wie motiviert sich die Studierenden trotz aller Widrigkeiten auf Grund von Corona mit den Originalobjekten auseinandergesetzt und mit welcher Kreativität sie sich in das Projekt eingebracht haben“, fasst Johanna Weiler zusammen.

Die Mehrheit der Seminar-Teilnehmer ${ }^{\star}$ innen hat das Projektteam auch bei der Konzeption der Ausstellung „17 Motive jüdischen Lebens“ unterstützt, die vom 4. November 2021 bis 16. Januar 2022 als Höhepunkt und Abschluss des Projektes im Schopenhauer-Studio der Universitätsbibliothek Frankfurt am Main zu sehen sein wird. An 17 Stationen können Besucher*innen die Motive und damit die facettenreiche deutsch-jüdische Kultur anhand historischer und zeitgenössischer Objekte erkunden und sind an vielen Stellen eingeladen, auch selbst aktiv $\mathrm{zu}$ werden. 
Das Projekt ist eingebunden in das bundesweite Festjahr \#2021JLID, das der Verein „321-2021: 1700 Jahre jüdisches Leben in Deutschland e.V.“ mit seiner Geschäftsstelle in Köln organisiert und koordiniert. Der Verein hat das interdisziplinäre Studien- und Ausstellungsprojekt der Bibliothek durch seine finanzielle Förderung ermöglicht.

\section{Weitere Informationen im Internet:}

- Projekt-Website: https://17motive.uni-frankfurt.de

- Instagram: https://www.instagram.com/frankfurter_dinge

- Festjahr: https://2021jlid.de

- Bildmaterial für die Presse: https://www.ub.uni-frankfurt.de/ausstellung/ 17motive_bilder.html

Presse-Besichtigung: mit Terminvereinbarung am 02.11. oder 03.11.2021.

\section{Weitere Informationen:}

Dr. Kerstin von der Krone, Sammlungsleitung Judaica- und Hebraica, und Johanna Weiler, Projekt 17 Motive

Universitätsbibliothek J. C. Senckenberg

Bockenheimer Landstraße 134-138 | 60325 Frankfurt am Main

Tel.: (069) 79839665 | E-Mail: 17motive@ub.uni-frankfurt.de

\section{Kontakt für Pressefragen allgemein:}

Bernhard Wirth, Stabsstelle Ausbildung und Öffentlichkeitsarbeit

Universitätsbibliothek J. C. Senckenberg

Bockenheimer Landstraße 134-138 | 60325 Frankfurt am Main

Tel.: (069) 79839223 | E-Mail: b.wirth@ub.uni-frankfurt.de 